\title{
Studies on Leaf Surface Lipid of Tobacco IV. Comparisons of Epicuticular Alkane Composition in Nicotiana Species
}

\author{
Kenji Gamou and Nobumaro KaWASHIMA \\ Utsunomiya Tobacco Experiment Station, The Japan Tobacco \& Salt Public Corporation, \\ 1900 Idei, Oyama-shi, Tochigi 323, Japan
}

Received August 8, 1980

\begin{abstract}
Alkane composition was determined on the leaves of 52 Nicotiana species and 2 horticultural species. All Nicotiana spcies except $N$. glauca contained substantial amounts of branched (iso- and anteiso-) alkanes. Nicotiana species were divided roughly into six groups by their resemblance on alkane composition.

This classification system was correlated to the botanical classification.

The evolution of Nicotiana species was discussed on the basis of alkane composition.
\end{abstract}

The use of chemical constituents of plants as an aid to their classification is now a familiar concept. The use of epicuticular wax in plant taxonomy would seem to advantageous in view of the almost universal occurrence of these coating and the fact that the wax is extracellular and almost certainly an end product insulated from the regular, essential metabolic functions of the plant. Above all, hydrocarbon mixture is very amenable to extract and rapid analysis by gas chromatography, and its very complexity serves as a positive advantage in providing a taxomomic "finger print." Attempting to test this approach, Eglinton and his colleagues examined the leaf waxes of closedy related genera of the subfamily Sempervivoideae (Crassulaceae). ${ }^{1,2)}$ In another study, Borges del Castillo et al. examined representatives of the families Podocarpaceae, Araucaraceae and Cupressaceae. ${ }^{3)}$ These results showed that there are some correlation between carbon number distribution and botanical classification. Since the above mentioned pioneering studies, many studies on the epicuticular wax from different species have been conducted $^{4}$ and the data are still accumulating. ${ }^{5,6\rangle}$

On the other hand, additional investigations with a limited range of plants have shown that factors such as growing conditions and age of tissue can also influence the distribution of wax components. ${ }^{79)}$ Although these results showed some difficulties for use of alkane composition in plant systematics, it is still an useful marker to distinguish plant species by the alkane composition, taking extent of the variation in consideration.

Nicotiana species have frequently used for introduction of disease resistant character into commercial tobacco plants by breeding. Since epicuticular wax may serve to preserve the water balance and to prevent the mechanical damage or fungal and insect attack, it is of interest to study the components of epicuticular wax in Nicotiana species. This paper deals with the comparative studies on epicuticular alkane composition in Nicotiana species.

\section{MATERIALS AND METHODS}

Seeds of Nicotiana species were derived from selfpollinated flowers at the Iwata Tobacco Experiment Station. Plants were grown in a green house in 1978 and 1979 repeatedly. All leaves of 5 plants, from every stalk position appeared on the ground, were taken at 3 to 4 months after seeding, when the respective plants flowered. In the case of some species which were not flowered under the conventional green house condition, samples were taken at 4 months after seeding.

Leaf surface lipid was extracted by a short time dipping of the leaves into chloroform according to a previous report. ${ }^{10)}$ After concentration of the extract 
in vacuo, alkane fraction was separated by a silica gel column according to a previous method. ${ }^{9}$ Alkane components were analyzed by $\mathrm{GC}$ on a $3 \mathrm{~m}$ column of $1 \%$ OV 101. Each GC peak of typical samples was identified by GC-MS and co-chromatography with an authentic sample.

\section{RESULTS}

The present experiment used 52 out of 64 Nicotiana species and 2 horticultural species ( $N$. affinis and $N$. sandelae). The plant names and relative composition of their alkane constituents are shown in Table I. Alkane components of the most species distributed into those which has carbon skelton number from 27 to 34 .

The components are roughly divided into 2 groups, one is branched such as iso (2-methyl) and anteiso (3-methyl) series and the other is normal series. Since the 2 groups are biosynthesized from different precursors, ${ }^{11)}$ respective analysis of the data for each group may be better way for species classification.

It has been known among various plants that N. tabacum, a commercial tobacco plant, is a species which contains branched alkane constituents in unusual high concentration. ${ }^{12)}$ Similar to $N$. tabacum, all Nicotiana species except for $N$. glauca had a substantial proportion of branched alkanes, and this may be a characteristic feature of Nicotiana species. In a previous paper, ${ }^{9}$ ' we reported that composition of alkanes especially for the branched components in $N$. tabacum was versatile with leaf age and cultivation circumstances. Nevertheless, the alkane composition seems to be an useful marker for classification, taking the compositional fluctuation in consideration. To serve for analysis of the data, degree of annual difference of the alkane composition of typical species are parallely shown in Table I as 1978 and 1979 data.

As reported on many higher plants, normal series of alkane components in Nicotiana species also distributed mostly in those with odd number carbon skelton, especially in $\mathrm{C}_{29}$, $\mathrm{C}_{31}$ and $\mathrm{C}_{33}$. In respect to these 3 components, Nicotiana species may be divided into 4 groups, where the first group consists of $n-\mathrm{C}_{31} / n-\mathrm{C}_{29}>2$, $2>n-\mathrm{C}_{31} / n-\mathrm{C}_{33}>0.5$, and $n-\mathrm{C}_{33} / n-\mathrm{C}_{29}>2(n-\mathrm{A})$, the second group $n-\mathrm{C}_{31} / n-\mathrm{C}_{29}>2, n-\mathrm{C}_{31} / n-\mathrm{C}_{33}$ $>2$ and $2>n-\mathrm{C}_{33} / n-\mathrm{C}_{29}>0.5(n-\mathrm{B})$, the third group $2>n-\mathrm{C}_{31} / n-\mathrm{C}_{29}>0.5, n-\mathrm{C}_{31} / n-\mathrm{C}_{33}>2$ and $n-\mathrm{C}_{33} / n-\mathrm{C}_{29}<0.5(n-\mathrm{C})$, and the fourth group $n$ $\mathrm{C}_{31} / n-\mathrm{C}_{29}>2, n-\mathrm{C}_{31} / n-\mathrm{C}_{33}<0.5$ and $n-\mathrm{C}_{33} / n-\mathrm{C}_{29}$ $>2(n-\mathrm{D})$. The results are also shown in Table I.

All species except for $N$. glauca in Paniculatae section belong to $n$-A. N. glauca was a special species in 52 Nicotiana species so far examined in all, and contained about $90 \%$ of $n-\mathrm{C}_{31}$ to total alkane content.

Species in Tomentosae section belong to either $n$-A or $n$-B. We classified $N$. tabacum cv. $\mathrm{MC}$ to be $n-\mathrm{B}$ in this experiment, but further expriments using 23 cultivars showed that these cultivars belong to either $n$-A or $n$-B.

All 3 species in Repandae section, $N$. nudicaulis in Nudicaulis section, $N$. wigandeoides in Undulata section and N. sylvestris in Alatae section belong to $n$-A.

Although $N$. rustica in Rusticae section showed annual difference in alkane composition, we tentatively classified it to be $n$-B taking average number between 1978 and 1979 data. $N$. trigonophylla in Trigonophyllae section, $N$. longiflora and $N$. plumbaginifolia in Alatae section and $N$. bigelovii in Bigelovianae section belong to $n-\mathrm{B}$.

In Sauveolentes species, 10 out of 17 species showed $n$-A and 5 species showed $n$-B. $N$. simulans may be $n$-A. $N$. goodspeedii showed a characteristic feature which does not contain $n-\mathrm{C}_{33}$.

As above mentioned, 39 out of 52 species in Nicotiana showed either $n$-A or $n$-B character. Although $n$-A and $n-\mathrm{B}$ were classified in the present data analysis, it was occasionally hard to distinguish the group of some species which were on around the border of $n-\mathrm{A}$ and $n-\mathrm{B}$. However, the above species could be clearly distinguished from the other two groups, $n$-C and $n$-D.

The third group, $n$-C, is consisted of 2 out of 3 species in Undulatae section and 2 out of 4 species in Acuminatae section. Another 2 
species in Acuminatae section showed characteristic features for respective species, but we would like to join these 2 species into $n-\mathrm{C}$ because of their relatively high content in $n-\mathrm{C}_{29}$ component. $N$. clevelandii in Bigelovinae section belong to this group.

The species which belong to $n$-D were only detected in Alatae section, where 6 including 2 horticultural species ( $N$. affinis and $N$. sandelae) out of 9 Alatae species belong to $n-\mathrm{D}$.

In regard to branched alkane composition, Nicotiana species may be divided into 4 groups, where the first group consists of iso- $\mathrm{C}_{31} /$ iso- $\mathrm{C}_{29}$ $>2$ and $2>$ anteiso- $\mathrm{C}_{32}$ /anteiso- $\mathrm{C}_{30}>0.5(b-\mathrm{A})$, the second group iso- $\mathrm{C}_{31} /$ iso- $\mathrm{C}_{29}>2$ and $8>$ anteiso- $\mathrm{C}_{32} /$ anteiso- $\mathrm{C}_{30}>2 \quad(b-\mathrm{B})$, the third group anteiso- $\mathrm{C}_{32} /$ anteiso- $\mathrm{C}_{30}<0.5$ regardless with iso- $\mathrm{C}_{31} /$ iso- $\mathrm{C}_{29}(b-\mathrm{C})$, and the fourth group iso- $\mathrm{C}_{31}$ /iso- $\mathrm{C}_{29}>8$ and anteiso- $\mathrm{C}_{32}$ /anteiso- $\mathrm{C}_{30}$ $>8(b-\mathrm{D})$.

In the case where the species showed annual difference in the grouping, for example in $N$. knightiana or $N$. rustica, the average number of the two samples was taken for tentative decision to which group the species belong to.

Five species in Paniculatae section except for $N$. glauca, belong to $b$-A. N. rustica, $N$. tabacum, $N$. trigonophylla, 3 out of 9 species in Alatae section, 5 out of 17 species in Sauveolentes section also belong to $b$-A.

The second group, $b-\mathrm{B}$, is consisted of all three species in Repandae section, $N$. nudicaulis in Nudicaulis section, and 9 out of 17 species in Sauveolentes section.

Contrary to the above two groups, the third group, $b-\mathrm{C}$, is consisted of species in more restricted sections, that is, 2 out of 3 species in Undulatae section, all 2 species in Bigelovinae section and all 4 species in Acuminatae section. As an exception, N. glutinosa in Tomentosae section belong to this group.

As to the fourth group, $b-\mathrm{D}, 6$ species in Alatae section, which classified as $n-\mathrm{D}$, belong to this group. In addition to this, 3 species in Sauveolentes section also showed the similar character.

Although the above grouping is not rigid but still fluctual in some extent, the present results may show that the classification of Nicotiana species by alkane composition has some correlation with the botanical classification.

\section{DISCUSSION}

In an authorized book "The Genus Nicotiana,"13! Goodspeed proposed an evolution scheme of Nicotiana species (Table II). Consideration of the present data on the evolutional aspect may be of interest, because each species seems to have its characteristic alkane composition or species in the same section have similar alkane composition.

$N$. rustica, which is being cultivated as a commercial tobacco in USSR, is known to be evolved after ancient hybridization of $N$. paniculata and $N$. undulata, ${ }^{13)}$ and showed an intermediate character in alkane composition between both progenitors. Previously, Dyson and Hervin ${ }^{16)}$ reported that hybrid plants of Cypressus species produced an intermediate alkane comstitution of their parent species. Suspecting from the results, alkane composition in $N$. rustica would be a reflection of its parent progenetors.

According to Goodspeed, 5 species in Tomentosae section (except for N. kawakamii which was a new species descovered in 1968) might be evolved from a pre-subgenic aggregate, pre-Tabacum. Although there are some species gap one another, these species showed generous similarity in alkane composition.

As described in Results, N. tabacum cultivars showed one of those characters which were $n$-A or $n$-B and $b-\mathrm{A}$ or $b$-B. Since these cultivars are the products after many times breedings not only in $N$. tabacum but also in some cases with the other Nicotiana species, we could not decide which cultivar is the typical $N$. tabacum. Unfortunately, it has not yet been detected wild $N$. tabacum in spite of enormous survey. Nevertherless, either of $N$. tabacum cultivar showed similarity in alkane composition with those in Tomentosae section. $N$. tabacum is known to be arisen after hybridization of $N$. sylvestris and $N$. tomentosiformis. ${ }^{14,15}$ ) Although N. sylvestris is botani- 


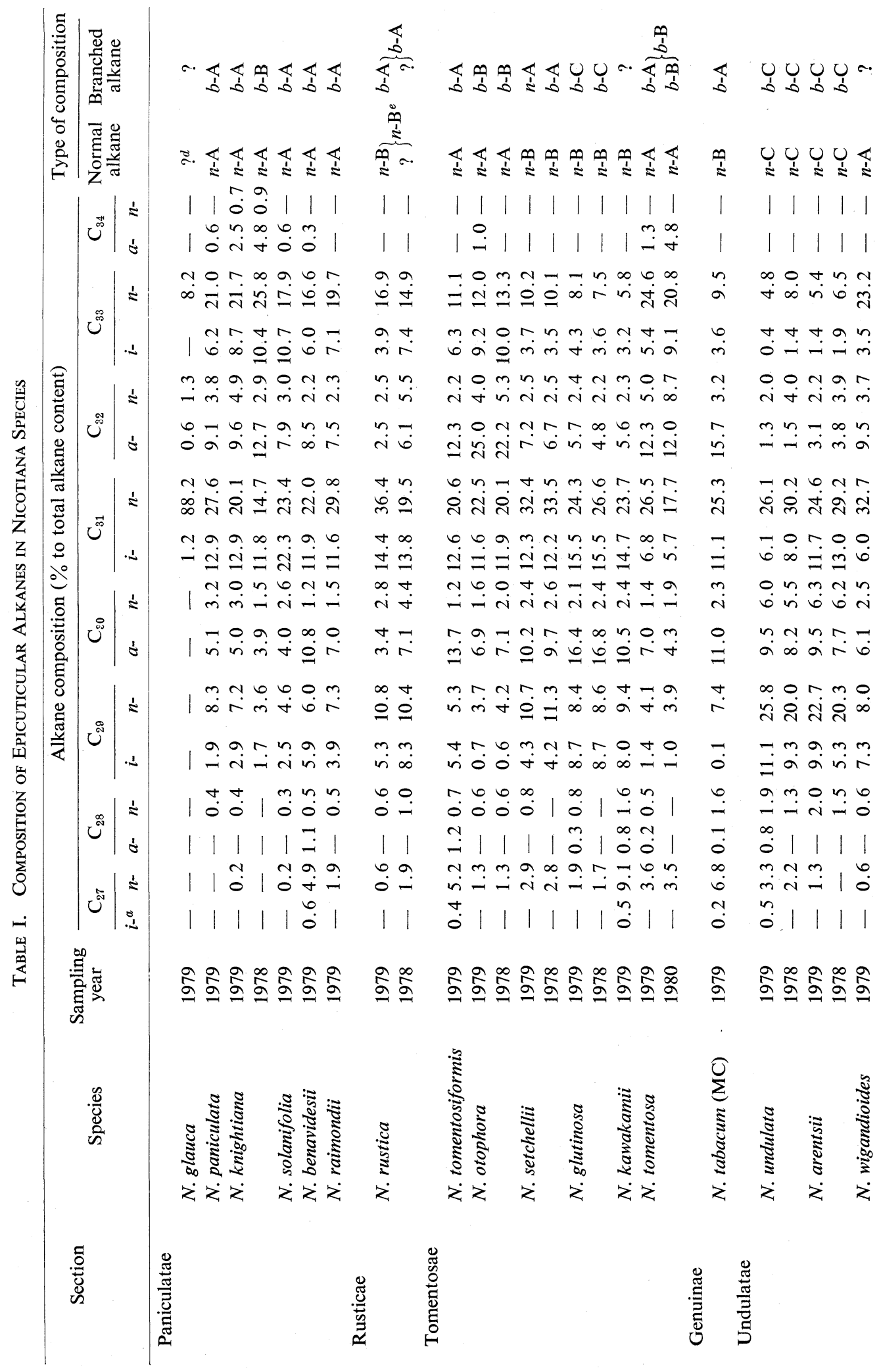




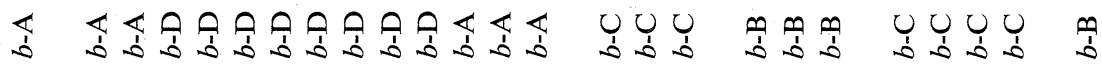

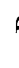

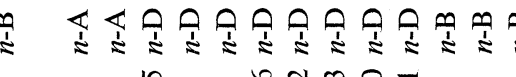

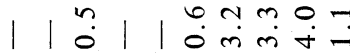

* 60 Nán n no

貓

$\infty$ a t rorma a $6 m \infty t$

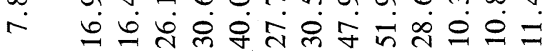

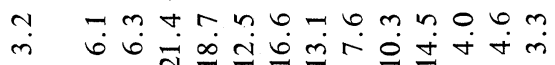

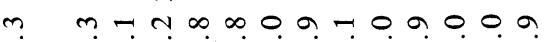

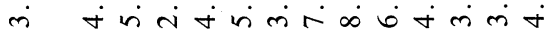

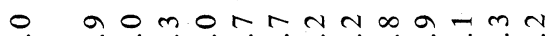

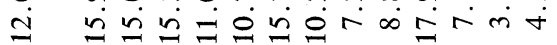

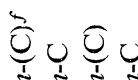

$\frac{1}{2}=$

\section{$+$}

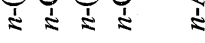

0

T.

om

- mo

111

.

จ่ กี่

กอก

in 6 in

ar $m$

$=\infty 0^{\circ}$

ㄱ.

$n \mathrm{Nm}$

in in

$\pm 0$

$m \backsim, n$

ro.

त 9 b

No

Ð

$\dot{m} \dot{r}$

तi

$\infty \pi$ ?

r.

in $\infty$

-

त)

$\forall \dot{r} \ddot{~}$

$\dot{0}$

$\infty n+$

$\mathrm{AT}+$

แ $N \infty \mathrm{N}$

$\exists$ ন

ปั

$\infty \stackrel{\sim}{-}$

$6 m$ n -

$\forall \infty \nabla$

$\pm \infty$

$\infty \sim \infty$

$+m m$

$\infty$ in

$m-m n$

ले $\dot{m}$

$-\lambda \dot{~}$

$0-\mathrm{N}$

a $+\forall \infty$

¿드

$\infty \simeq$

๑ั

$\infty$

Nm

ஸึ่

$\stackrel{\infty}{+\infty} \dot{+}$

$-c \dot{ }$

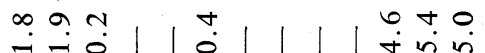

$+r \infty$

$1 \%$

ㄴ. $m$

$\therefore a \dot{0} \sim$

a N

I

o mon

$\dot{-i} \dot{\pi}$

N $m-m$

¿̊ं

111

$\stackrel{-\pi}{0}$

$+m \infty$

กั

$1 \tilde{0}$

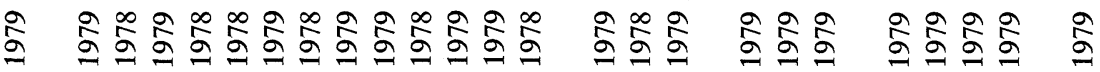
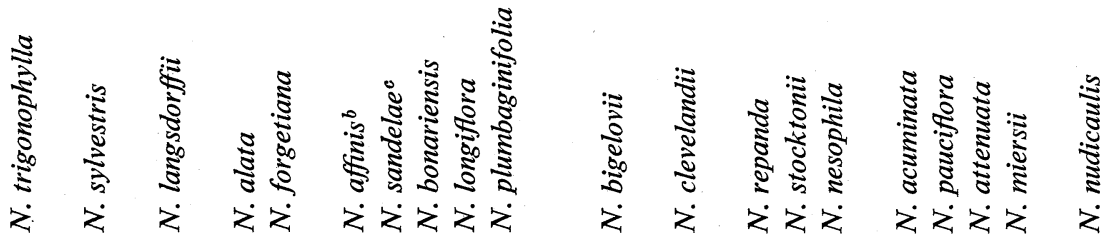

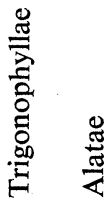

焉

䢘 


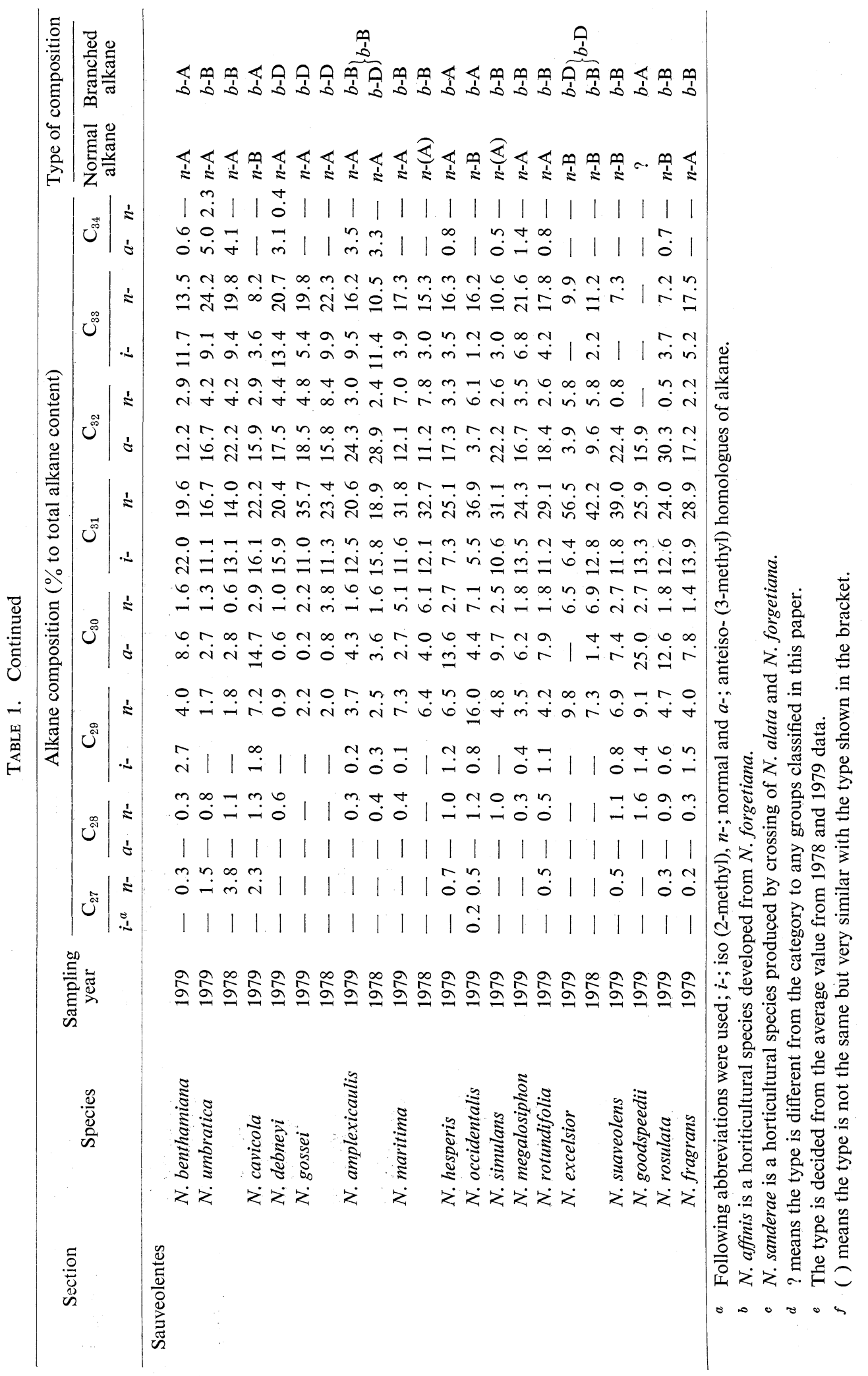


TAble II. The Evolutionary Scheme of Nicotiana Species Proposed by Goodspeed and the Classtfication by Alkane Composition

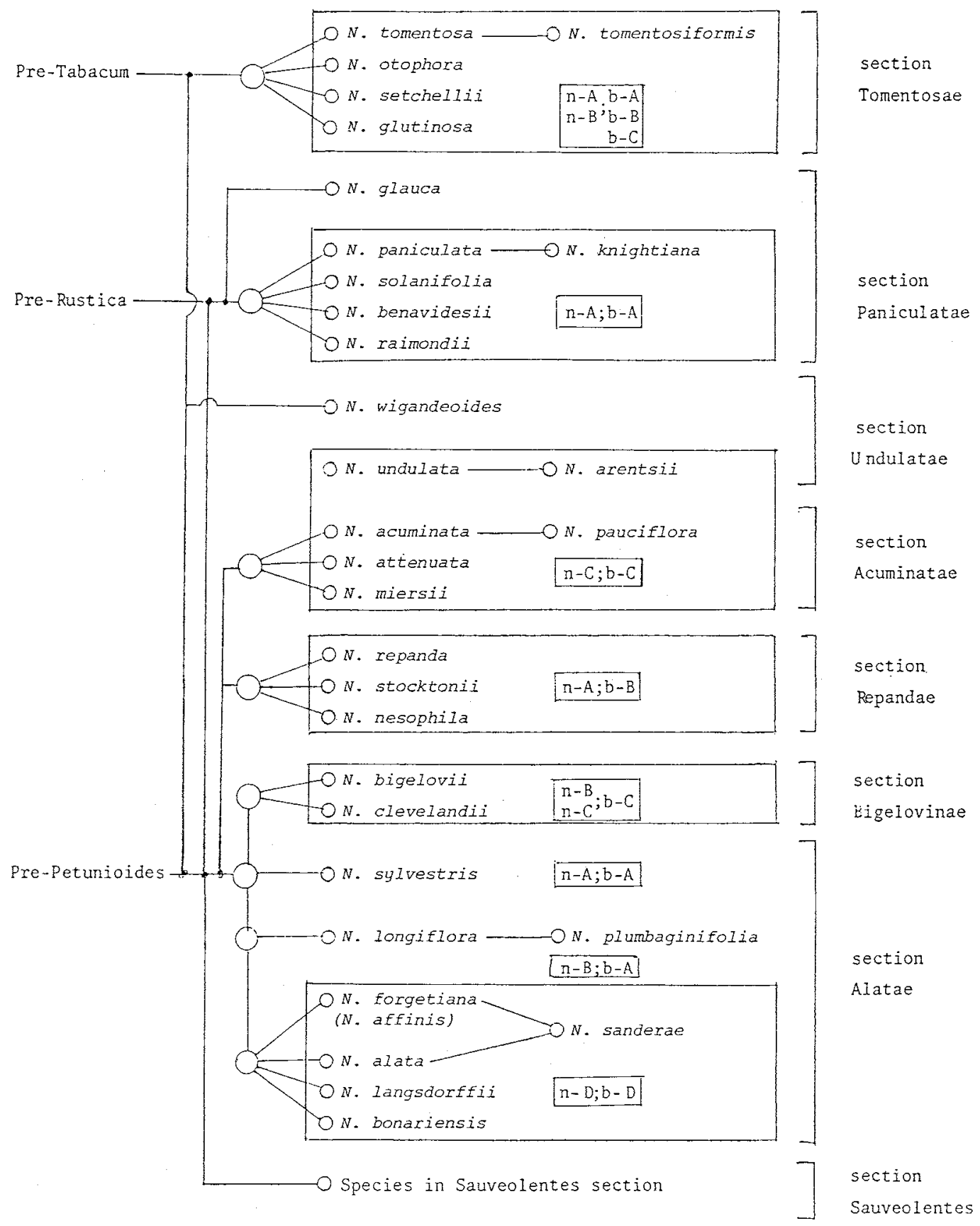


cally classified in Alatae section, it has also similar character in alkane composition with the species in Tomentosae section, but not with the majority of the Alatae species. Therefore it may be reasonable that $N$. tabacum has Tomentosae type alkane composition.

In Undulatae species, $N$. undulata and $N$. arentsii has similar alkane composition, while $N$. wigandeoides has quite different composition. Although these 3 species are botanically classified in the same section, $N$. undulata and $N$. arentsii were thought to be arisen after contact or pre-Rustica with pre-Petunioides aggregate, while $N$. wigandeoides was arisen after contact of pre-Tabacum and pre-Petunioides aggregate, and this may be the reason why $N$. wigandeoides has different alkane composition from the other two species. Goodspeed cited that $N$. wigandeoides is marked in similarities to Tomentosae section botanically, and the present result also showed that $N$. wigandeoides has similar alkane composition with Tomentosae species.

Species in Alatae section is thought to be products of pre-Petunioides aggregate. Above all, $N$. sylvestris $(n=12)$ would be evolved the most anciently from 12 paired pre-Alatae race. What next occurred was the chromosome reduction, and $N$. longiflora and $N$. plumbaginifolia would be arisen $(n=10)$. The final chromosome reduction in this section would be occurred producing another species in Alatae section $(n=9)$. Effect of the chromosome reduction might reflect to the alkane composition, as the species with $n=9$ has quite different alkane composition from the other species.

Species in Sauveolentes section so far examined in all showed very low relative contents in iso- $\mathrm{C}_{29}$. In this regard, these species are similar with Alatae species with $n=9$. Another chemotaxonomical similarity between the species in Sauveolentes and Alatae sections has been shown in the primary structure of Fraction 1 protein..$^{15)}$ These results may imply that either Sauveolentes or Alatae species were evolved from the same progenetor, pre-Petunioides, as Goodspeed proposed.

The present results showed close relation between alkane composition and evolutional situation of Nicotiana species. However, we are still afraid of that the compatibility is fortuitous because alkane composition is very flexible with the environmental condition where plants grow. To confirm the hypothesis that alkane composition has correlation with evolution of species, more precise experiments under restricted condition must be necessary and our study is going to this direction.

\section{REFERENCES}

1) G. Eglinton, A. G. Gonzalez, R. J. Hamilton and R. A. Raphael, Phytochem., 1, 89 (1962).

2) G. Eglinton, R. J. Hamilton, R. A. Raphael and A. G. Gonzalez, Nature, 193, 739 (1962).

3) J. Borges del Castillo, C. J. W. Brooks, G. Eglinton, R. J. Hamilton and P. Pellitt, Phytochem., 6, 391 (1967).

4) A.P. Tulloch, "Chemistry and Biochemistry of Natural Waxes," ed. by P. E. Kolattukudy, Elsevier, Amsterdam, p. 235 (1976).

5) G. Bianchi P. Avato, P. Bertorelli and G. Mariani, Phytochem., 17, 999 (1978).

6) A. P. Tulloch and L. L. Hoffman, Phytochem., 18, 267 (1979).

7) H. Stocker and H. Wanner, Phytochem., 14, 1919 (1975).

8) E. A. Baker, M. J. Bukovac and J. A. Flore, Phytochem., 18, 781 (1979).

9) K. Gamou and N.Kawashima, Agric. Biol. Chem., in press.

10) K. Gamou and N. Kawashima, Agric. Biol. Chem., 43, 2163 (1979).

11) P. E. Kolattukudy, Ann. Rev. Plant Physiol., 21, 163 (1970).

12) J. D. Mold, R. K. Stevens, R. E. Means and J. M. Ruth, Biochemistry, 2, 605 (1963).

13) T.H. Goodspeed, "The Genus Nicotiana," Chronica Botanica Co., USA, Chapter 13.

14) D. Kostoff, C. R. Acad. Sci. USSR, 18, 459 (1938).

15) N. Kawashima, Y. Tanabe and S. Iwai, Biochim. Biophys. Acta, 427, 70 (1976).

16) W. G. Dyson and G. A. Herbin, Phytochem., 7, 1339 (1968). 